\title{
Establishing criteria to initiate enteric outbreak investigations in British Columbia
}

\author{
Taylor $\mathbf{M}^{1^{*}}$, Galanis $\mathrm{E}^{1,2}$ \\ British Columbia Centre for Disease Control, Vancouver, BC \\ School of Population and Public Health, University of British Columbia, Vancouver, BC \\ Corresponding author: marsha.taylor@bccdc.ca
}

\section{Abstract}

Objective: To establish and evaluate criteria to initiate provincial enteric outbreak investigations based on characteristics of alerts, clusters and successful outbreak investigations.

Methods: We tracked all enteric disease alerts and clusters reported to the British Columbia Centre for Disease Control (BCCDC) in 2011 and 2012. Information was collected on etiology, number of cases, geographic spread, dates reported, and method of notification. Actions were classified as no further action, review/report or investigation. Outbreak investigation outcome was classified as solved/not solved. 2011 data were used to identify characteristics of alerts and clusters more likely to lead to outbreak investigations and of solved outbreaks to establish criteria. Criteria for initiating an outbreak investigation were evaluated retrospectively using 2011 data and then implemented in 2012.

Results: In 2011, 251 alerts/clusters of enteric diseases were reported. Fourteen (5.6\%) led to an outbreak investigation and nine (64.3\%) of the outbreaks were solved. Analyzing the data retrospectively, criteria were identified from the alerts and clusters that led to outbreak investigations and successful outbreak investigations: pathogen specificity, timely notification, a common source or event, and multi-regional outbreaks or outbreaks reported by other agencies. After applying these criteria prospectively in 2012 , we took action on a smaller proportion of the 244 alerts and clusters $(32.0 \%$ compared to $44.6 \%$ in 2011 ) and $66.7 \%$ of them were solved (compared to $64.3 \%$ in 2011).

Conclusion: Continued evaluation will identify whether this will improve outbreak investigations and use of resources in British Columbia.

\section{Introduction}

The goal of enteric disease outbreak investigations (includes foodborne, waterborne, and those spread through person-to-person or animal contact) is to identify the source of illness in order to control the outbreak, limit the number of cases and identify recommendations to prevent future outbreaks. Identifying a source also provides information on high-risk products, modes of transmission and effective interventions (1). However, the proportion of solved enteric disease outbreaks (where a source is identified) is low $(2,3)$. In British Columbia (BC), we have previously taken steps to improve outbreak investigations by determining enteric diseases to be routinely investigated, using standard case investigation forms, and maintaining collaborative relationships between epidemiologists and laboratory staff.

Outbreak investigations are expensive and resource intensive. Responding to alerts that do not develop into outbreaks (false alarms) and unsuccessful investigations lead to a waste of scarce public health resources. Improving the ability to solve and control outbreaks by identifying and focusing on those which have the greatest chance of a successful outcome so that resources could be used most appropriately would be valuable to public health professionals. In addition, use of a consistent and transparent approach to initiate outbreak investigations creates a standard practice and increases partner confidence in the process.

In BC, population 4.5 million (4), the British Columbia Centre for Disease Control (BCCDC) is responsible for provincial surveillance and the coordination of outbreak investigations that span multiple local health regions. 
BCCDC also participates in national outbreak investigations and provides assistance for outbreaks in single local health regions when requested. In 2009 and 2010, there was an average of 225 enteric disease alerts and clusters reported each year to BCCDC; 10 of 19 (52.6\%) outbreak investigations undertaken were solved. During this time there were no criteria for initiating an investigation and the decision to investigate was made based on investigators' experience and judgment. To our knowledge, there are no criteria used in Canada to initiate enteric outbreaks investigations.

The objectives of this work were to: 1 ) identify the characteristics of enteric alerts and clusters that we investigated; 2) identify the characteristics of successful enteric outbreak investigations in 2011 to establish criteria to initiate provincial enteric outbreak investigations; and 3) evaluate those criteria and compare outbreak investigations in 2011 and 2012.

\section{Methods}

A systematic process was used to track all incoming notifications of enteric disease alerts and clusters from a variety of sources between January 1, 2011, and December 31, 2012. Alerts with two or more cases caused by the same pathogen and clusters where two or more unrelated cases with similar illness were epidemiologically linked were included. The only exception to this was botulism, where one case was considered an outbreak, and hepatitis $A$ that was excluded because it is investigated by a separate group at BCCDC. All clusters and alerts had to be reported to or identified by BCCDC epidemiologists. Sources of reported clusters included local health regions, the BC Public Health Microbiology and Reference Laboratory, other provinces/territories, federal agencies (e.g., Canadian Food Inspection Agency, Public Health Agency of Canada), and international agencies (e.g., U.S. state Departments of Health).

The source of alerts was the provincial reportable diseases database. BCCDC runs an automated aberration detection system on reportable diseases on a weekly basis. The data inputs include five years of reportable disease at genus level and five years of lab information (at serotype/species level and phage type ( $S$. Enteritidis, S. Heidelberg) or PFGE (PFGE) (E. coli O157, S. Typhimurium, S. sonnei). A time series analysis identifies weekly aberrations (alerts) taking seasonal and temporal trends into consideration. Alerts are produced when the disease count is significantly above that expected over time periods of $7,14,21$ and 28 days, at provincial or local levels.

Information on alerts and clusters was collected in a prospective fashion at the time of notification. Each alert or cluster that met the inclusion criteria was captured and information was collected on etiology, including level of subtyping, number of cases, geographic spread, initial date reported, previous knowledge of alert or cluster, and initial method of notification. Furthermore, the action taken by a BCCDC epidemiologist for each alert or cluster was documented. Actions were classified into three categories:

- No further action taken.

- Review/Report - We used existing reportable disease data or laboratory data to assess for commonalities or we reported information to public health partners but did not require further investigation.

- Outbreak investigation-We used a coordinated approach to investigate or respond to an outbreak. This usually involved requesting and reviewing case exposure information from local health regions and using additional investigative methods (e.g., re-interviews, site investigations, environmental sampling) to identify and control the source.

Of the alerts and clusters that led to an outbreak investigation, we classified them as solved if a source was identified based on an analytical study, laboratory detection of the pathogen in the source or a combination of case exposure information and trace back to a potential common source.

The 2011 data were used to establish criteria for initiating an outbreak investigation based on information about the pathogen, number of cases, time period between notification and most recently reported case using the earliest of available dates (report date, collection date or onset date), geography and available exposure information. The criteria were established by comparing alerts and clusters that led to an outbreak investigation to those that did not and outbreak investigations which were solved to those that were unsolved. The 2011 data 
were used to retrospectively evaluate the criteria. The criteria were used throughout 2012 and data were used to prospectively evaluate the criteria by comparing the number and proportion of alerts and clusters that were investigated, solved and controlled compared to 2011 to assess the impact of implementing the criteria. MS Excel $B$ was used for data collection and analysis. Chi-square tests were calculated using SAS and Epi Calc 2000 to compare proportions and medians. A p-value of $<0.05$ was considered significant.

\section{Results}

\section{Assessment of alerts, clusters and investigations}

In 2011, a total of 251 alerts and clusters were reported. Fourteen (5.6\%) led to an outbreak investigation (Table 1). Alerts and clusters which led to an outbreak investigation were significantly different from those we did not investigate; they had a higher proportion with PFGE $(p=<0.001)$, they were more likely to involve multiple local health regions $(p=0.002)$ and a higher proportion were reported by other agencies $(p=<0.001)$. The median number of cases was higher, although not significantly so and the time between most recent case and notification was similar (Table 1).

Table 1. Characteristics of enteric disease alerts and clusters reported to BCCDC by type of action taken, BC, 2011 ( $\mathrm{N}=\mathbf{2 5 1})$

\begin{tabular}{|l|l|l|l||c|}
\hline $\begin{array}{l}\text { Characteristics at } \\
\text { time of notification }\end{array}$ & $\begin{array}{l}\text { Conducted } \\
\text { outbreak } \\
\text { investigation } \\
(\mathbf{N}=\mathbf{1 4})\end{array}$ & $\begin{array}{l}\text { Reviewed data } \\
\text { and/or reported } \\
\text { to partners } \\
\mathbf{( N = 9 8 )}\end{array}$ & $\begin{array}{l}\text { No further } \\
\text { action taken }\end{array}$ & P value \\
\hline Proportion of total & $5.6 \%$ & $39.0 \%$ & $55.4 \%$ & $\begin{array}{l}\text { Not } \\
\text { applicable }\end{array}$ \\
\hline $\begin{array}{l}\text { Number (\%) with } \\
\text { pulsed field gel } \\
\text { electrophoresis } \\
\text { (PFGE) }\end{array}$ & $6(42.8 \%)$ & $13(13.3 \%)$ & $4(2.9 \%)$ & $<0.001$ \\
\hline $\begin{array}{l}\text { Median number of } \\
\text { cases; range }\end{array}$ & $6(1-30)$ & $3(1-106)$ & $3(1-68)$ & 0.630 \\
\hline $\begin{array}{l}\text { Median number (and } \\
\text { range) of days } \\
\text { between notification } \\
\text { and most recently } \\
\text { reported case }\end{array}$ & $4(1-30)$ & $3(1-47)$ & Not assessed & 0.710 \\
\hline $\begin{array}{l}\text { Number ( \%) with } \\
\text { multiple local health } \\
\text { regions involved }\end{array}$ & $5(35.7 \%)$ & $22(22.4 \%)$ & $15(10.8 \%)$ & 0.002 \\
\hline $\begin{array}{l}\text { Number ( \%) notified } \\
\text { by other agency }\end{array}$ & $10(71.4 \%)$ & $14(14.3 \%)$ & 0 & $<0.001$ \\
\hline
\end{tabular}

In 2011, nine (64.3\%) of the outbreaks were solved. Solved outbreaks were more likely to involve a rare pathogen/subtype, be notified by another agency and have less than two weeks between most recent case and 
notification. None of the differences were statistically significant. A common event/location and indication of source at time of notification were only identified in solved investigations (Table 2).

Table 2. Characteristics of solved and unsolved enteric disease outbreak investigations, BC, 2011 $(\mathrm{N}=14)$

\begin{tabular}{|l|c|c|}
\hline Characteristics at time of notification & $\begin{array}{c}\text { Solved } \\
\text { outbreak* } \\
\text { (N=9) }\end{array}$ & $\begin{array}{c}\text { Unsolved } \\
\text { outbreak** } \\
\text { (N=5) }\end{array}$ \\
\hline Proportion of total & $64.3 \%$ & $35.7 \%$ \\
\hline $\begin{array}{l}\text { Pathogen Specificity } \\
\text { Number and \% with pulsed field gel electrophoresis } \\
\text { (PFGE) }\end{array}$ & $2(22.2 \%)$ & $4(80.0 \%)$ \\
\hline $\begin{array}{l}\text { Number and \% with a rare pathogen/subtype } \\
\text { Timeliness }\end{array}$ & $6(66.7 \%)$ & $2(40.0 \%)$ \\
\hline $\begin{array}{l}\text { Median number (and range) of days between notification } \\
\text { and most recently reported case }\end{array}$ & $3(1-30)$ & $16(1-21)$ \\
\hline $\begin{array}{l}\text { Number ( \%) with less than two weeks between onset, } \\
\text { report, collection date of last case and notification }\end{array}$ & $8(88.9 \%)$ & $2(40.0 \%)$ \\
\hline Median number of cases; (range) & $5(1-30)$ & $6(1-9)$ \\
\hline Number ( \%) with multiple local health regions involved & $3(33.3 \%)$ & $2(40.0 \%)$ \\
\hline Number ( \%) notified by other agency & $7(77.8 \%)$ & $3(60.0 \%)$ \\
\hline Number (\%) with indication of source & $4(44.4 \%)$ & 0 \\
\hline Number (\%) with common event/location & $4(44.4 \%)$ & 0 \\
\hline
\end{tabular}

* Solved outbreaks included: diarrhetic shellfish poisoning (DSP) associated with cooked mussels (5); botulism associated with watermelon jelly; E. coli O157:H7 associated with veal liver; S. Kingabwa associated with pet lizards; S. Enteritidis associated with eggs; two norovirus investigations in a resort; S. Agbeni associated with a food handler (6); S. Infantis associated with a food handler.

** Unsolved outbreaks included: one Campylobacter investigation in the community; one E. coli O157:H7 investigation in the community; and three $S$. Enteritidis investigations in the community.

\section{Establishment of criteria}

Pathogen-specific criteria were established to identify alerts and clusters to review and investigate (Table 3). The criteria include a minimum number of cases based on the pathogen and specificity, a maximum timeframe between most recent case occurrence and notification, a requirement for multi-regional distribution or epidemiological link or investigations initiated by other agencies. 
Figure 1. Criteria for the review of provincial enteric disease alerts and clusters ${ }^{1}$

\begin{tabular}{|c|c|c|}
\hline $\begin{array}{l}\text { Minimum number of } \\
\text { cases }\end{array}$ & \multicolumn{2}{|c|}{$\begin{array}{l}\text { Pathogen with common serotype/species with further typing or rare } \\
\text { serotype/species (e.g., E. coli O157 with the same PFGE, Listeria } \\
\text { monocytogenes): Range of } 2-5 \text { cases } \\
\text { Pathogen with common genus/serotype/species without further typing (e.g., S. } \\
\text { Heidelberg without molecular typing, Campylobacter jejuni, Giardia): Range of } \\
10-20 \text { cases } \\
\text { AND }\end{array}$} \\
\hline Maximum timeframe & \multicolumn{2}{|c|}{$\begin{array}{l}\text { Earliest of available dates (report date, collection date or onset date) of most } \\
\text { recent case within } 14 \text { days of notification date } \\
\text { AND }\end{array}$} \\
\hline Geographic distribution & \multicolumn{2}{|l|}{ Multi-regional or unknown } \\
\hline \multicolumn{3}{|c|}{$\begin{array}{l}\text { OR } \\
\text { - Single case of botulism OR } \\
\text { - Any number of cases with earliest of available dates (report date, collection date or onset date) within } \\
14 \text { days of notification date with an epidemiological link to each other, a food or common source OR } \\
\text { - Any number of cases associated with investigations initiated by other jurisdictions }\end{array}$} \\
\hline & Review & $\begin{array}{l}\text { EXCLUDE: } \\
\text { Alerts or clusters } \\
\text { associated with } \\
\text { travel/immigration, a }\end{array}$ \\
\hline & $\begin{array}{l}\text { REPORT/ INVESTIGATE: } \\
\text { Level of investigation is based on the } \\
\text { pathogen and available information }\end{array}$ & $\begin{array}{l}\text { data entry error or } \\
\text { previously investigated }^{2}\end{array}$ \\
\hline
\end{tabular}

${ }^{1}$ Note: The criteria are subject to change over time as part of ongoing work and evaluation.

${ }^{2}$ If new information became available on the same cases or new cases were reported, the criteria would be applied using the new level of information.

The number of cases among alerts/cluster investigated and solved outbreaks varied by pathogen. Specificity of the pathogen assisted with both identification and solving of outbreaks. The level of specificity required varied based on the pathogen; while common pathogens required serotype or PFGE (e.g., E. coli O157 and S.

Enteritidis), rarer pathogens or syndromes did not (e.g., DSP). We set thresholds for the minimum number of cases required at notification considering levels of specificity which varied from two (e.g., listeriosis) to 20 (e.g., giardiasis). For pathogens with greater pathogen specificity, the minimum number of cases required was lower.

Among the 2011 successful outbreak investigations, seven $(77.8 \%)$ had less than seven days between the most recent case onset and notification (data not shown) and eight $(88.9 \%)$ had less than fourteen days. We selected a 14-day period criterion to include most of the solvable outbreaks while acknowledging the need for timely action.

To meet the BCCDC provincial mandate, our criteria required that alerts and clusters affect multiple local health regions or have an unknown geographic spread at time of notification. 
An indication of source at notification and epidemiological links between cases or to a common event or location were common among solved outbreaks. These characteristics likely also indicate a temporal clustering of cases and potentially more timely notification.

\section{Evaluation of criteria}

The 14 outbreak investigations from 2011 were evaluated using the criteria. Eleven (78.6\%) outbreaks met the criteria for further investigation. Of the three that did not, one was conducted in a single local health region and two had most recent cases occur earlier than 14 days prior to notification (28 and 30 days). The investigation in the single local health region was solved. Neither of the outbreaks with a longer time period was solved, suggesting that our criteria would appropriately exclude such events. In addition, two events that were not investigated in 2011 would have met the criteria for initiating an investigation. Both were caused by Salmonella (one with a rare serotype, one with a common serotype but matching PFGE) and had four cases reported over a seven- and a fourteen-day period. Retrospective review of laboratory and available case information did not identify commonalities.

In 2012, the criteria were implemented prospectively. A total of 244 alerts and clusters were reported. Only three $(1.2 \%)$ led to outbreak investigations; two were solved. In 2012, fewer alerts and clusters led to review/report (30.7\% compared to $39.0 \%$ ) and a larger proportion had no further action taken $(68.0 \%$ compared to $55.4 \%)$ than in 2011. No known outbreaks were missed.

Throughout 2012, adjustments were made to the criteria because new laboratory methods (e.g., change in shiga toxin detection) were implemented, pathogens not previously considered were identified, and some thresholds within criteria were found to be too sensitive.

\section{Discussion}

Criteria that identified outbreaks which were more likely to be solved included characteristics of pathogen specificity, timeliness and known linkages between cases or a possible source at the time of notification. The use of our criteria in 2012 led to a $21.3 \%$ decrease in the number of clusters and alerts that were reviewed. This was largely due to no longer reviewing alerts affecting a single local health region (48.4\%). This likely led to a concomitant reduction in use of resources both provincially and locally as less time was spent locating, reviewing, sharing, and centrally analyzing data. The retrospective evaluation of our criteria identified two clusters in 2011 that met our criteria but were not investigated, likely because of limited case information to indicate relatedness.

Some of our criteria are compatible with those used in other jurisdictions and some are unique.

In Minnesota, an evaluation of Salmonella outbreaks suggested greatest success with three cases notified to the state public health agency within seven days of each other (7). In a similar evaluation of $E$. coli O157:H7 outbreaks, the shorter the time period in which the first two isolates were received, the greater the likelihood was of solving the outbreak. Although our measure of time was different, this suggests that having a sufficient number of cases reported close in time to each other and recently notified to a public health agency helps identify solvable outbreaks. It may also indicate a common event or location. Evaluations focused on a single pathogen have demonstrated greater success in investigating Salmonella and E. coli O157:H7 PFGE clusters with four or more and three or more cases, respectively $(7,8)$. For PFGE clusters of Salmonella $(\mathrm{N}=3)$ and shiga toxin producing $E$. coli $(\mathrm{N}=3)$, the number of cases needed in $\mathrm{BC}$ was similar to what Rounds et al. found $(7,8)$. Use of pathogen subtyping such as PFGE has been shown to be of value in detecting outbreaks, particularly dispersed outbreaks $(2,7)$. Incorporation of pathogen specificity information into our criteria is important for detection of provincial outbreaks which are often more dispersed.

Other similar evaluations have employed a retrospective review of clusters using laboratory data or outbreaks reported to an electronic surveillance system or summarized by the jurisdiction (7-9). Our method was unique in that it employed prospective data collection of information available at the time of notification to document decisions and set criteria which could be used and useful at this point in the assessment process. Our proportion of solved investigations is higher than or similar to other published examples $(2,3,7-9)$ likely because we included only clusters and alerts we classified as outbreaks after initial steps were taken to review them, or based on our classification of solved. 
There are a number of limitations to this study. A prospective method provided fewer years of data for assessment. Because we were aware of our process we had the ability to modify our actions over time. This awareness may have led to a bias in the selection of clusters and alerts more likely to be solved prior to criteria establishment. Although this affects our assessment of the proportion of outbreaks solved, it should not affect the determination of the criteria for initiation of an investigation. Our criteria will likely identify outbreaks associated with acute point sources, continuous sources and person-to-person transmission in a common location. It is possible that they will miss small and/or intermittent outbreaks with cases that occur over a longer period of time. We will require additional methods of notification to detect these and will rely on pathogen subtyping to identify links. Our criteria may be relevant to other jurisdictions but the levels within the criteria may need to be adjusted based on availability of case information, willingness and resources available to investigate, and laboratory subtyping capacity.

Future efforts are underway to further develop and refine these criteria. The criteria need to be flexible so that changes can easily be made over time. Ongoing data collection and analysis are planned in order to assess the proportion of investigations solved and controlled over time, to allow for updates to the criteria and to identify whether outbreaks are missed.

We undertook this work in order to improve outbreak investigation processes and outcomes. This process has improved our understanding of the elements that can lead to successful outbreak investigations. Over time we hope to improve the proportion of outbreaks solved and controlled to decrease the morbidity associated with enteric illness and improve the use of resources.

\section{References}

(1) Gould LH, Walsh KA, Vieira AR, Herman K, Williams IT, Hall AJ, Cole D. Surveillance for foodborne disease outbreaks—United States, 1998-2008. MMWR. 2013;62(2): 1-34

(2) Murphee R, Garman K, Phan Q, Everstine K, Gould LH, Jones, TF. Characteristics of foodborne disease outbreak investigations conducted by Foodborne Diseases Active Surveillance Network (FoodNet) sites, 2003-2008. Clin Infect Dis. 2012;54(S5):S498-503.

(3) Jones TF, Rosenberg L, Kubota K, Ingram LA. Variability among states in investigating foodborne disease outbreaks. J Food Prot. 2013;10(1):69-73.

(4) Government of British Columbia. BC Stats-Population Estimates. Available from: http://www.bcstats.gov.bc.ca/StatisticsBySubject/Demography/PopulationEstimates.aspx

(5) Taylor M, McIntyre L, Ritson M, Stone J, Bronson R, Bitzikos O, Rourke W, Galanis E, Outbreak Investigation Team. Outbreak of diarrhetic shellfish poisoning associated with mussels, British Columbia, Canada. Mar Drugs. 2013;15(5):1669-76.

(6) Taylor M, Brisdon S, Jeyes J, Stone J, Embree G, Paccagnella A, Hoang L, Galanis E. Salmonella enterica servoar Agbeni, British Columbia, Canada, 2011. Emerg Infect Dis. 2012;18(9):1542-3.

(7) Rounds JM, Hedberg C, Meyer S, Boxrud DJ, Smith KE. Salmonella enterica PFGE clusters, Minnesota, USA, 2001-2007. Emerg Infect Dis. 2010; 16(11):1678-85.

(8) Rounds JM, Boxrud DJ, Jawahir SL, Smith KE. Dynamics of Escherichia coli O157:H7 outbreak detection and investigation, Minnesota 2000-2008. Epidemiol Infect. 2012;140(8):1430-8.

(9) Gaulin C, Currie A, Gravel G, Hamel M, LeBlanc MA, Ramsay D, Bekal S. Summary of 11 years of enteric outbreak investigation and criteria to initiate an investigation, Province of Quebec, 2002 through 2012. JFP. In press 2014.

\section{Acknowledgements}

The authors would like to acknowledge representatives from Fraser Health Authority, Interior Health Authority, Northern Health Authority, Vancouver Coastal Health Authority and Vancouver Island Health Authority for their support of this work and collaboration during outbreak investigations and the clinical microbiology laboratories in $\mathrm{BC}$ and the BC Public Health Microbiology and Reference Laboratory responsible for diagnosis of enteric infections. 


\section{Conflict of interest}

No conflicts of interests to declare.

\section{Funding}

NA 